03

\title{
Решение задач рассеяния волн телами, имеющими изломы границы, и фракталоподобными телами вращения
}

\author{
(C) А.Г. Кюркчан ${ }^{1,2,3}$, С.А. Маненков ${ }^{1, \uparrow}$, Н.И. Смирнова ${ }^{1}$ \\ ${ }^{1}$ Московский технический университет связи и информатики, \\ 111024 Москва, Россия \\ ${ }^{2}$ Фрязинский филиал Института радиотехники и электроники им. В.А. Котельникова РАН, \\ 141190 Фрязино, Московская обл., Россия \\ ${ }^{3}$ Центральный научно-исследовательский институт связи, \\ 111141 Москва, Россия \\ ๑ e-mail: mail44471@mail.ru
}

Поступила в редакцию 28.11.2018 г.

В окончательной редакции 28.11.2018 г.

Принята к публикации 09.01.2019 г.

На основе метода продолженных граничных условий предложена методика, позволяющая моделировать характеристики рассеяния, в том числе усредненные по углам ориентации, для тел практически любой геометрии. Приведен ряд примеров решения задач дифракции на фракталоподобных телах вращения. Корректность метода подтверждена при помощи проверки выполнения оптической теоремы для различных тел и путем сравнения с результатами расчетов, полученных методом дискретных источников.

DOI: $10.21883 /$ OS.2019.05.47651.348-18

\section{Введение}

Методика, предложенная в работе [1], распространена на трехмерный случай. Исследование характеристик рассеяния волн телами сложной геометрии представляет большой интерес в таких областях, как астрофизика, оптика, акустика и др. Подобного рода исследования выполняют, как правило, с использованием метода $T$-матриц [2]. Этот метод весьма популярен, что подтверждает, в частности, обзор [3], в котором приведены ссылки более, чем на 250 публикаций за 2015-17 гг. Популярность метода $T$-матриц объясняется во многом тем, что с использованием этого метода можно сравнительно легко выполнять такую важную, например, в астрофизике процедуру, как усреднение характеристик рассеяния телом по углам его ориентации относительно падающего поля. Однако, как было показано ранее в наших работах (например, [2,4]), традиционный метод $T$-матриц корректен для достаточно узкого класса геометрий рассеивателя.

Метод $T$-матриц, предложенный П. Уотерменом в начале 60-х годов XX века [5], получил впоследствии широкое распространение в радиофизике, акустике, астрофизике и других областях науки. Его популярность объясняется простотой и удобством вычисления важных в приложениях характеристик рассеяния компактных объектов. Ранее в наших работах было показано, что традиционный метод $T$-матриц корректен лишь для так называемых релеевских тел [2]. Таким образом, представляет интерес распространение техники этого метода на задачи рассеяния волн телами достаточно произвольной формы.
Под методом $T$-матриц (МТМ) принято понимать процедуру нахождения матрицы, связывающей коэффициенты разложения по некоторому (обычно угловому или сферическому) базису поля, рассеянного какимлибо объектом при падении на него плоской волны [1], называемые коэффициентами рассеяния (см. ниже). Элементы $T$-матрицы не зависят от угла падения первичной волны и определяются лишь геометрией рассеивателя и видом краевых условий на его границе. Это позволяет легко выполнять важные в ряде приложений [3] операции усреднения характеристик рассеяния по углам падения первичной волны (углам ориентации рассеивателя). Традиционный (классический) вариант метода $T$-матриц [5], как и его недавно разработанные модифицированные варианты $[1,4,6,7]$, применимы, как показано в [1] и более ранних работах авторов, к решению задач дифракции лишь на рассеивателях с аналитической границей. В то же время в астрофизике, радиолокации и других областях весьма востребованы задачи рассеяния волн телами с изломами границы, тонких экранах и др. Таким образом, представляет большой интерес распространение техники $T$-матриц на задачи рассеяния волн телами с неаналитической границей. В настоящей работе изложен подход, основанный на методе продолженных граничных условий (МПГУ) [2,8].

Как известно [9], в оптическом поле интенсивность электромагнитного поля можно приближенно выразить через одну комплексную скалярную функцию, т.е. векторная задача дифракции может быть приближенно заменена скалярной. В настоящей работе рассмотрена трехмерная скалярная задача дифракции плоской волны на телах вращения, в частности, на фракталоподобных телах со сложным поперечным сечением. 


\section{1. Вывод основных соотношений}

Перейдем к изложению предлагаемого подхода. Рассмотрим дифракцию на компактном препятствии в виде тела вращения, ограниченном кусочно-гладкой поверхностью $S$. Пусть на границе $S$ рассеивателя выполняется условие Дирихле

$$
\left.U\right|_{S}=0 .
$$

Здесь $U(\mathbf{r})=U^{0}(\mathbf{r})+U^{1}(\mathbf{r})$ - полное поле, где $U^{0}(\mathbf{r})-$ падающее, а $U^{1}(\mathbf{r})$ - рассеянное (вторичное) поле. В качестве падающего поля рассмотрим плоскую волну

$U^{0}(\mathbf{r})=\exp \left(-i k r\left(\sin \theta \sin \theta_{0} \cos \left(\varphi-\varphi_{0}\right)+\cos \theta \cos \theta_{0}\right)\right)$,

где $\theta_{0}, \varphi_{0}$ - углы падения волны, $k-$ волновое число среды, окружающей тело, $(r, \theta, \varphi)$ - сферические координаты. На бесконечности выполнено условие излучения для рассеянного поля:

$$
\frac{\partial U^{1}}{\partial r}+i k U^{1}=o\left(\frac{1}{r}\right), \quad r \rightarrow \infty .
$$

Как известно (например, [2]), для дифракционного поля $U^{1}(\mathbf{r})$ имеет место следующее представление:

$$
\int_{S}\left\{U(\mathbf{r}) \frac{\partial G_{0}\left(\mathbf{r}, \mathbf{r}^{\prime}\right)}{\partial n^{\prime}}-\frac{\partial U\left(\mathbf{r}^{\prime}\right)}{\partial n^{\prime}} G_{0}\left(\mathbf{r}, \mathbf{r}^{\prime}\right)\right\} d s^{\prime}=U^{1}(\mathbf{r}),
$$

в котором

$$
G_{0}\left(\mathbf{r} ; \mathbf{r}^{\prime}\right)=\frac{1}{4 \pi} \frac{\exp \left(-i k\left|\mathbf{r}-\mathbf{r}^{\prime}\right|\right)}{\left|\mathbf{r}-\mathbf{r}^{\prime}\right|}=\frac{1}{4 \pi} \frac{e^{-i k R}}{R}
$$

- фундаментальное решение уравнения Гельмгольца (функция Грина свободного пространства) в трехмерном случае. С учетом условия (1) представление (4) примет следующий вид:

$$
\int_{S} \frac{\partial U\left(\mathbf{r}^{\prime}\right)}{\partial n^{\prime}} G_{0}\left(\mathbf{r} ; \mathbf{r}^{\prime}\right) d s^{\prime}=-U^{1}(\mathbf{r})
$$

где $\partial / \partial n^{\prime}$ означает дифференцирование в направлении внешней нормали к поверхности $S$. Потребовав в соответствии с МПГУ выполнения условия (1) на поверхности $S_{\delta}$, расположенной в $\mathbf{R}^{3} \backslash \bar{D}$, где $D$ - область внутри $S[2,8]$, с использованием (6) получим следующее интегральное уравнение Фредгольма I рода с гладким ядром:

$$
\left.\int_{S} \frac{\partial U\left(\mathbf{r}^{\prime}\right)}{\partial n^{\prime}} G_{0}\left(\mathbf{r} ; \mathbf{r}^{\prime}\right) d s^{\prime}\right|_{S_{\delta}}=\left.U^{0}(\mathbf{r})\right|_{S_{\delta}}
$$

Отметим, что чаще всего в качестве $S_{\delta}[2,8]$ выбирают поверхность, охватывающую $S$ и отстоящую от нее на некоторое достаточно малое расстояние $\delta$, т.е. рассматривают эквидистантную поверхность. Пусть рассеиватель представляет собой тело вращения, уравнение границы $S$ которого задается в параметрическом виде

$$
\left\{\begin{array}{l}
x=\rho(t) \cos \varphi, \\
y=\rho(t) \sin \varphi, \\
z=z(t),
\end{array}\right.
$$

где $(\rho, \varphi, z)$ - цилиндрические координаты. При этом параметр $t$ изменяется на интервале $\left[0, t_{\max }\right]$. Тогда уравнения смещенной поверхности $S_{\delta}$ записываются следующим образом:

$$
\left\{\begin{array}{l}
x=\rho_{\delta}(t) \cos \varphi, \\
y=\rho_{\delta}(t) \sin \varphi, \\
z=z_{\delta}(t),
\end{array}\right.
$$

где $\rho_{\delta}(t)=\rho(t)+n_{\rho}(t) \delta, z_{\delta}(t)=z(t)+n_{z}(t) \delta$, причем $n_{\rho}$ и $n_{z}-$ координаты нормали к поверхности тела $S$. Теперь с использованием обозначения

$$
\left.\frac{1}{2} \frac{\partial U\left(\mathbf{r}^{\prime}\right)}{\partial n^{\prime}}\right|_{S} d s^{\prime} \equiv J\left(t^{\prime}, \varphi^{\prime}\right) d t^{\prime} d \varphi^{\prime}
$$

уравнение (7) примет вид

$$
\left.\frac{1}{2 \pi} \int_{0}^{2 \pi} \int_{0}^{t_{\max }} J\left(t^{\prime}, \varphi^{\prime}\right) \frac{e^{-i k R}}{R} d t^{\prime} d \varphi^{\prime}\right|_{S_{\delta}}=\left.U^{0}(\mathbf{r})\right|_{S_{\delta}}
$$

где

$$
R=\sqrt{\rho_{\delta}(t)^{2}+\rho\left(t^{\prime}\right)^{2}-2 \rho_{\delta}(t) \rho\left(t^{\prime}\right) \cos \left(\varphi-\varphi^{\prime}\right)+\left(z_{\delta}(t)-z\left(t^{\prime}\right)\right)^{2}} .
$$

Далее, с учетом фурье-разложений

$$
\begin{gathered}
\left.U^{0}(\mathbf{r})\right|_{S_{\delta}}=U^{0}(t, \varphi)=\sum_{s=-\infty}^{\infty} U_{s}^{0}(t) e^{i s \varphi}, \\
J\left(t^{\prime}, \varphi^{\prime}\right)=\sum_{s=-\infty}^{\infty} I_{s}\left(t^{\prime}\right) e^{i s \varphi^{\prime}}, \\
\left.\frac{e^{-i k R}}{R}\right|_{S_{\delta}} \equiv K\left(t, t^{\prime}, \tau\right)=\sum_{s=-\infty}^{\infty} K_{s}\left(t, t^{\prime}\right) e^{i s \tau},
\end{gathered}
$$

(где $\tau=\varphi-\varphi^{\prime}$ ) уравнение (11) сводится к следующей системе одномерных интегральных уравнений (СИУ):

$$
\int_{0}^{t_{\max }} I_{s}\left(t^{\prime}\right) K_{s}\left(t, t^{\prime}\right) d t^{\prime}=U_{s}^{0}(t), \quad s=\overline{-Q, Q},
$$

$Q$ - верхний предел суммирования в соотношениях (12), и

$$
\begin{aligned}
K_{s}\left(t, t^{\prime}\right) & =\frac{1}{2 \pi} \int_{0}^{2 \pi} \frac{e^{-i k R\left(t, t^{\prime}, \tau\right)}}{R\left(t, t^{\prime}, \tau\right)} e^{-i s \tau} d \tau, \\
U_{s}^{0}(t) & =\frac{1}{2 \pi} \int_{0}^{2 \pi} U^{0}(t, \varphi) e^{-i s \varphi} d \varphi .
\end{aligned}
$$


Систему (13) можно решать, например, методом Крылова-Боголюбова. С этой целью представим неизвестные функции $I_{s}\left(t^{\prime}\right)$ в виде

$$
I_{s}\left(t^{\prime}\right) \cong \sum_{n=1}^{N} I_{n, s} \Phi_{n}\left(t^{\prime}\right), \quad s=\overline{-Q, Q}
$$

где $\Phi_{n}\left(t^{\prime}\right)$ - импульсные функции вида

$$
\Phi_{n}\left(t^{\prime}\right)=\left\{\begin{array}{ll}
1, & t \in\left[t_{n}-\Delta / 2, t_{n}+\Delta / 2\right], \\
0, & t \notin\left[t_{n}-\Delta / 2, t_{n}+\Delta / 2\right]
\end{array} .\right.
$$

Здесь $t_{n}=\frac{t_{\max }}{N}\left(n-\frac{1}{2}\right), n=\overline{1, N}, \Delta=\frac{t_{\max }}{N}-$ шаг сетки, $N-$ число базисных функций. Далее, подставив (15) в СИУ (13) и приравняв левую и правую части полученных равенств в точках коллокации, выбранных на поверхности $S_{\delta}$, получим следующие системы алгебраических уравнений относительно величин $I_{n, s}$ :

$$
\sum_{n=1}^{N} K_{m n, s} I_{n, s}=U_{m, s}^{0}, \quad s=\overline{-Q, Q}, \quad m=\overline{1, N},
$$

где матричные элементы и правые части вычисляются по формулам

$$
K_{n m, s}=\int_{t_{n}-\Delta / 2}^{t_{n}+\Delta / 2} K_{s}\left(t_{m}, t\right) d t, \quad U_{m, s}^{0}=U_{s}^{0}\left(t_{m}\right) .
$$

Отметим, что для эффективного вычисления матричных элементов в системе (17), т.е. величин $K_{s}\left(t_{m}, t^{\prime}\right)$, использовался алгоритм, приведенный в работе [10]. С целью ускорения вычислений мы находили внешние интегралы в формуле (18), используя адаптивную методику (позволяющую вычислять интегралы от быстро меняющихся функций), только для элементов матрицы, расположенных вблизи главной диагонали, а остальные матричные элементы вычисляли, используя квадратуру Гаусса с двумя узлами.

Правильность полученных результатов контролируется путем вычисления величины невязки в точках поверхности $S_{\delta}$, расположенных посередине между точками коллокации. Одним из критериев правильности полученных результатов также является оптическая теорема, которая записывается [11] в виде

$$
\begin{aligned}
\frac{k^{2} \sigma}{4 \pi} & \equiv \frac{1}{4 \pi} \int_{\Omega}\left|f\left(\theta, \varphi ; \theta_{0}, \varphi_{0}\right)\right|^{2} \sin \theta d \theta d \varphi \\
& =-\operatorname{Im} f\left(\theta=\theta_{0}, \varphi=\varphi_{0}\right),
\end{aligned}
$$

где $f\left(\theta, \varphi ; \theta_{0}, \varphi_{0}\right)$ - диаграмма рассеяния, выражение для которой приведено ниже. В качестве оценки точности выполнения оптической теоремы будем рассчитывать величину $\Delta_{o t}$, которая представляет собой относительную разность левой и правой части в формуле (19).
Для дальнейшего будет удобно переписать системы (17) в матричных обозначениях:

$$
\mathrm{K}_{s} \mathbf{I}_{s}=\mathbf{a}_{s}, \quad s=\overline{-Q, Q},
$$

где $\mathrm{K}_{s}=\left\|K_{m n, s}\right\|, \mathbf{a}_{s}=\left\|U_{m, s}^{0}\right\|, m, n=\overline{1, N}$. Найдя коэффициенты $I_{n, s}$ из систем (20) с учетом формул (6), $(10),(12)$ и (15), получим следующее представление для рассеянного поля:

$$
U^{1}(\rho, z)=-\sum_{s=-Q}^{Q} \sum_{n=1}^{N} I_{n, s} \int_{t_{n}-\frac{\Delta}{2}}^{t_{n}+\frac{\Delta}{2}} K_{s}\left(\rho, z, t^{\prime}\right) d t^{\prime} \cdot e^{i s \varphi},
$$

где

$$
K_{s}\left(\rho, z, t^{\prime}\right)=\frac{1}{2 \pi} \int_{0}^{2 \pi} \frac{e^{-i k R}}{R} e^{-i s \tau} d \tau,
$$

$$
R=\sqrt{\rho^{2}+\rho\left(t^{\prime}\right)^{2}-2 \rho \rho\left(t^{\prime}\right) \cos \tau+\left(z-z\left(t^{\prime}\right)\right)^{2}} .
$$

Перейдя к асимптотике выражения (21) при $r \rightarrow \infty$, получим представление для диаграммы рассеяния:

$$
\begin{aligned}
& f\left(\theta, \varphi, \theta_{0}, \varphi_{0}\right)=-\sum_{s=-Q}^{Q} \sum_{n=1}^{N} i^{s} I_{n, s} \\
& \quad \times \int_{t_{n}-\frac{\Delta}{2}}^{t_{n}+\frac{\Delta}{2}} J_{s}(k \rho(t) \sin \theta) \exp (i k z(t) \cos \theta) d t e^{i s \varphi} .
\end{aligned}
$$

Здесь $J_{s}(x)$ - цилиндрические функции Бесселя. В формуле (22) коэффициенты $I_{n, s}$ зависят от углов падения $\theta_{0}, \varphi_{0}$ плоской волны.

Рассмотрим вопрос о нахождении диаграммы рассеяния, усредненной по углам падения плоской волны (например, в случае равновероятного распределения углов падения $\left.\theta_{0}, \varphi_{0}\right)$ :

$$
\langle f(\theta, \varphi)\rangle=\frac{1}{4 \pi} \int_{0}^{2 \pi} \int_{0}^{\pi} f\left(\theta, \varphi, \theta_{0}, \varphi_{0}\right) \sin \theta_{0} d \theta_{0} d \varphi_{0} .
$$

Вначале приведем стандартную схему нахождения усредненной диаграммы при помощи метода $T$-матриц. Всюду вне сферы, описанной вокруг $S$, в соответствии с теоремой сложения [12] имеем

$$
\begin{aligned}
& \frac{\exp (-i k R)}{R}=-i k \sum_{p=0}^{\infty} \sum_{q=-p}^{p}(2 p+1) \\
& \times \frac{(p-q) !}{(p+q) !} h_{p}^{(2)}(k r) j_{p}\left(k r^{\prime}\right) P_{q}^{p}(\cos \theta) P_{q}^{p}\left(\cos \theta^{\prime}\right) e^{i q\left(\varphi-\varphi^{\prime}\right)} .
\end{aligned}
$$


С учетом определения величин $K_{s}\left(r, \theta, t^{\prime}\right)$ из (24) получим

$$
\begin{aligned}
& K_{s}\left(r, \theta, t^{\prime}\right)=-i k \sum_{p=|s|}^{\infty}(2 p+1) \\
& \quad \times \frac{(p-s) !}{(p+s) !} h_{p}^{(2)}(k r) j_{p}\left(k r^{\prime}\left(t^{\prime}\right)\right) P_{p}^{s}(\cos \theta) P_{p}^{s}\left(\cos \theta^{\prime}\left(t^{\prime}\right)\right),
\end{aligned}
$$

где $j_{p}(x)-$ сферические функции Бесселя, $h_{p}^{(2)}(x)-$ сферические функции Ганкеля второго рода, $P_{p}^{s}(x)-$ присоединенные функции Лежандра, $r^{\prime}(t), \theta^{\prime}\left(t^{\prime}\right)$ - сферические координаты точки на контуре осевого сечения тела. Теперь из (21) и (25) получим

$$
\begin{aligned}
& U^{1}(\mathbf{r})=i k \sum_{s=-\infty}^{\infty} \sum_{p=|s|}^{\infty}(2 p+1) \frac{(p-s) !}{(p+s) !} h_{p}^{(2)}(k r) P_{p}^{s}(\cos \theta) \\
& \quad \times e^{i s \varphi} \sum_{n=1}^{N} I_{n, s} \int_{t_{n}-\frac{\Delta}{2}}^{t_{n}+\frac{\Delta}{2}} j_{p}\left(k r^{\prime}(t)\right) P_{p}^{s}\left(\cos \theta^{\prime}(t)\right) d t .
\end{aligned}
$$

Перепишем соотношение (26) в стандартном для МТМ виде:

$$
U^{1}(\mathbf{r})=\sum_{s=-\infty}^{\infty} \sum_{p=|s|}^{\infty} c_{p, s} h_{p}^{(2)}(k r) P_{p}^{s}(\cos \theta) e^{i s \varphi},
$$

где

$$
\begin{aligned}
c_{p, s}= & i k(2 p+1) \frac{(p-s) !}{(p+s) !} \sum_{n=1}^{N} I_{n, s} \\
& \times \int_{t_{n}-\frac{\Delta}{2}}^{t_{n}+\frac{\Delta}{2}} j_{p}\left(k r^{\prime}(t)\right) P_{p}^{s}\left(\cos \theta^{\prime}(t)\right) d t .
\end{aligned}
$$

В матричных обозначениях будем иметь

$$
\begin{gathered}
\mathbf{c}_{s}=\mathrm{J}_{s} \mathbf{I}_{s}=\left(\mathrm{J}_{s} \mathrm{~K}_{s}^{-1}\right) \mathbf{a}_{s}=\mathrm{T}_{s} \mathbf{a}_{s}, \quad \mathbf{J}_{s}=\left\|J_{s, p n}\right\|, \\
J_{s, p m}=i k(2 p+1) \frac{(p-s) !}{(p+s) !} \int_{t_{n}-\frac{\Delta}{2}}^{t_{n}+\frac{\Delta}{2}} j_{p}\left(k r^{\prime}(t)\right) P_{p}^{s}\left(\cos \theta^{\prime}(t)\right) d t .
\end{gathered}
$$

Здесь $\mathrm{T}_{s}=\mathrm{J}_{s} \mathrm{~K}_{s}^{-1}$ и есть $T$-матрица для $s$-й азимутальной гармоники. В случае, когда первичным полем является плоская волна (2), распространяющаяся под углом $\theta_{0}$ к оси $O z$,

$$
U_{s}^{0}=\exp \left(-i k z_{\delta}(t) \cos \theta_{0}\right)(-i)^{s} J_{s}\left(k \rho_{\delta}(t) \sin \theta_{0}\right) .
$$

При $k r \rightarrow \infty$ имеем

$$
h_{p}^{(2)}(k r) \cong i^{p+1} \frac{e^{-i k r}}{k r}, \quad U^{1}(\mathbf{r}) \cong \frac{e^{-i k r}}{k r} f(\theta, \varphi) .
$$

Таким образом, из (27) получим

$$
f(\theta, \varphi)=\sum_{s=-\infty}^{\infty} \sum_{p=|s|}^{\infty} c_{p s} i^{p+1} P_{p}^{s}(\cos \theta) e^{i s \varphi}
$$

Как отмечалось во введении, во многих приложениях представляет интерес усредненная по углам ориентации тела диаграмма рассеяния. Усреднение по углам ориентации (с учетом (23)) приводит к соотношению

$$
\langle f(\theta)\rangle=\sum_{p=0}^{\infty}\left\langle c_{p 0}\right\rangle i^{p+1} P_{p}(\cos \theta),
$$

в котором

$$
\left\langle\mathbf{c}_{0}\right\rangle=\mathrm{T}_{0}\left\langle\mathbf{a}_{0}\right\rangle,
$$

где $\left\langle\mathbf{a}_{0}\right\rangle=\left\|\left\langle U_{m, 0}^{0}\right\rangle\right\|, m=\overline{1, N}$. Например, в случае равновероятного распределения улов ориентации (углов падения первичной плоской волны) получим

$$
\begin{aligned}
\left\langle U_{m, 0}^{0}\right\rangle= & \frac{1}{2} \int_{0}^{\pi} J_{0}\left(k \rho_{\delta}\left(t_{m}\right) \sin \theta_{0}\right) \\
& \times \exp \left(i k z_{\delta}\left(t_{m}\right) \cos \theta_{0}\right) \sin \theta_{0} d \theta_{0} .
\end{aligned}
$$

Усредненную диаграмму можно также находить непосредственно из формулы (22). Тогда

$$
\langle f(\theta)\rangle=-\sum_{n=1}^{N}\left\langle I_{n, 0}\right\rangle \int_{t_{n}-\frac{\Delta}{2}}^{t_{n}+\frac{\Delta}{2}} J_{0}(k \rho(t) \sin \theta) \exp (i k z(t) \cos \theta) d t,
$$

где коэффициенты $\left\langle I_{n, 0}\right\rangle$ находятся из системы (20) (при $s=0)$, в которой правые части заменяются на величины (36).

Приведем усредненную диаграмму сферы. Как известно, диаграмма рассеяния сферы радиуса $a$ в случае условия Дирихле на ее поверхности имеет вид

$$
\begin{aligned}
& f\left(\theta, \varphi, \theta_{0}, \varphi_{0}\right)=-i \sum_{p=0}^{\infty} \sum_{q=-p}^{p}(2 p+1) \\
& \times \frac{(p-q) !}{(p+q) !} \frac{j_{p}(k a)}{h_{p}^{(2)}(k a)} P_{p}^{q}(\cos \theta) P_{p}^{q}\left(\cos \theta_{0}\right) \exp \left(i q\left(\varphi-\varphi_{0}\right)\right) .
\end{aligned}
$$

Вычисляя интеграл (23), получим

$$
\langle f(\theta, \varphi)\rangle=-i \frac{j_{0}(k a)}{h_{0}^{(2)}(k a)} .
$$

Таким образом, усредненная диаграмма сферы, как и следовало ожидать, постоянна. 


\section{2. Результаты расчетов}

В табл. 1 приведены результаты расчета модуля диаграммы рассеяния идеально отражающего конуса (на поверхности тела выполнены условия Дирихле) с сечением равностороннего треугольника со стороной $2 k a=20$ и идеального цилиндра с квадратным сечением со стороной $2 k a=20$. Плоская волна падала перпендикулярно оси вращения тела. Диаграмму рассеяния вычисляли при $\varphi=0$. Параметр $k \delta=10^{-3}$. Численные результаты, приведенные в таблице, получены двумя методами: при помощи модифицированного метода дискретных источников (ММДИ) и МПГУ. Отметим, что ММДИ не может быть непосредственно применен к задаче дифракции на телах, имеющих изломы границы (таких как конус и цилиндр), поэтому для решения задачи при помощи ММДИ проводилась аппроксимация контура осевого сечения тела гладким контуром (например, [13]). В частности, при решении задачи дифракции

Таблица 1. Сравнение результатов, полученных при помощи ММДИ и МПГУ

\begin{tabular}{c|c|c|c|c}
\hline Угол $\theta, \operatorname{deg}$ & \multicolumn{2}{|c|}{ Диаграмма конуса } & \multicolumn{2}{c}{ Диаграмма цилиндра } \\
\cline { 2 - 5 } & МПГУ & ММДИ & МПГУ & ММДИ \\
\hline 0 & 0.7575 & 0.7552 & 2.1862 & 2.2179 \\
60 & 4.7958 & 4.8092 & 11.227 & 11.235 \\
90 & 42.999 & 43.080 & 88.089 & 87.920 \\
150 & 3.9152 & 3.9191 & 4.1936 & 4.2130 \\
180 & 1.8440 & 1.8408 & 2.1862 & 2.2179 \\
240 & 1.2636 & 1.2600 & 3.5127 & 3.5558 \\
270 & 1.3481 & 1.3444 & 17.930 & 17.817 \\
330 & 12.127 & 12.146 & 1.8718 & 1.9515
\end{tabular}

Таблица 2. Точность выполнения оптической теоремы для разных геометрий

\begin{tabular}{l|c|c|c}
\hline \multicolumn{1}{c|}{ Форма тела } & \multicolumn{2}{|c|}{$\begin{array}{c}\text { Значения левой и правой } \\
\text { частей в формуле (19) }\end{array}$} & Значение \\
\hline Конус & 39.18898 & 39.18820 & $1.99 \cdot 10^{-5}$ \\
\hline Цилиндр & 83.49031 & 83.49032 & $2.82 \cdot 10^{-8}$ \\
\hline $\begin{array}{l}\text { Сечение в виде } \\
\text { снежинки Коха } \\
(1 \text { итерация) }\end{array}$ & 51.95889 & 51.95778 & $2.13 \cdot 10^{-5}$ \\
\hline $\begin{array}{l}\text { Сечение в виде } \\
\text { снежинки Коха } \\
\text { (2 итерация) }\end{array}$ & 60.02143 & 60.02200 & $9.59 \cdot 10^{-6}$ \\
\hline $\begin{array}{l}\text { Сечение в виде } \\
\text { кривой Серпинского } \\
(1 \text { итерация) }\end{array}$ & 45.92960 & 45.92939 & $4.68 \cdot 10^{-6}$ \\
\hline $\begin{array}{l}\text { Сечение в виде } \\
\text { кривой Серпинского } \\
(2 \text { итерация) }\end{array}$ & 40.27679 & 40.27682 & $7.30 \cdot 10^{-7}$ \\
\hline
\end{tabular}
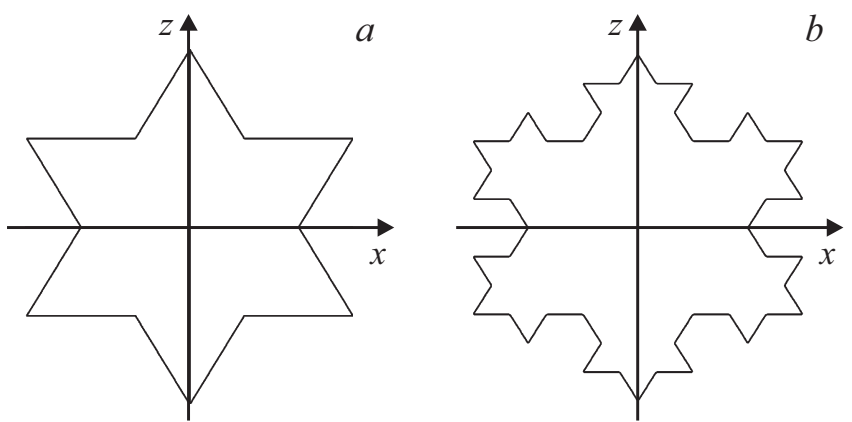

Рис. 1. Сечение тела в виде снежинки Коха: $a$ - первая итерация, $b-$ вторая итерация.
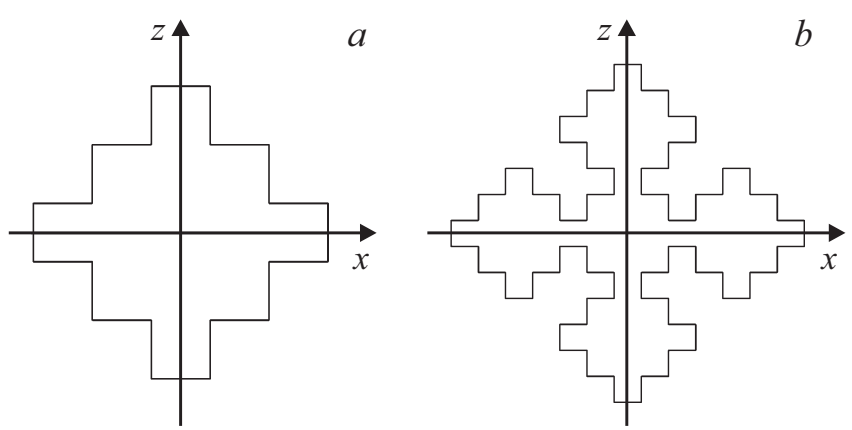

Рис. 2. Сечение тела в виде кривой Серпинского: $a-$ первая итерация, $b-$ вторая итерация.

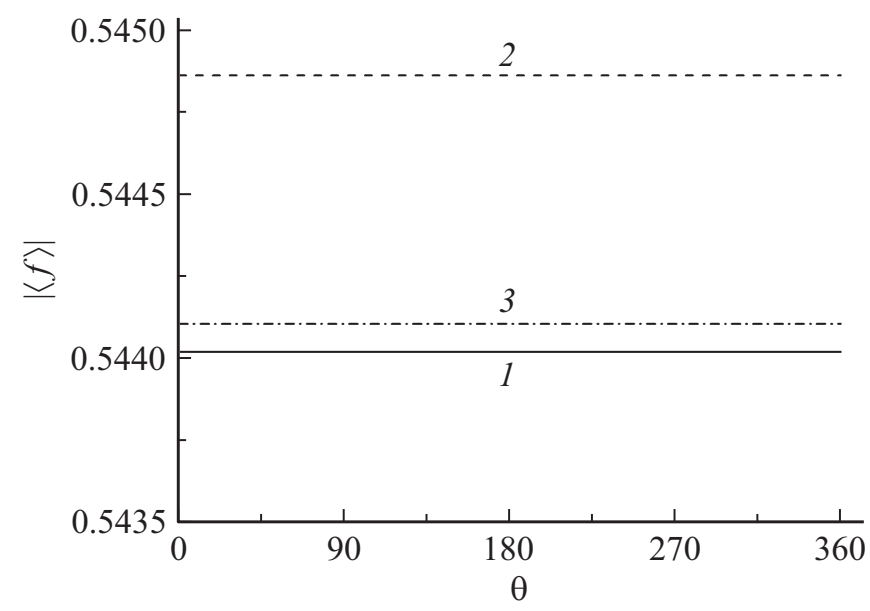

Рис. 3. Угловая зависимость усредненной диаграммы для сферы.

на цилиндре граница тела аппроксимировалась поверхностью суперэллипсоида вращения. Как видно из табл. 1, относительная разность результатов, полученных при помощи ММДИ и МПГУ, не превосходит $5 \cdot 10^{-3}$ в случае дифракции на конусе и $4 \cdot 10^{-2}$ для цилиндра.

В табл. 2 приведены результаты проверки точности выполнения оптической теоремы для указанных выше тел (конуса и цилиндра), а также для тела вращения с сечением в виде снежинки Коха и с сечением в виде кривой Серпинкого (рассматривались две итерации) [14]. 


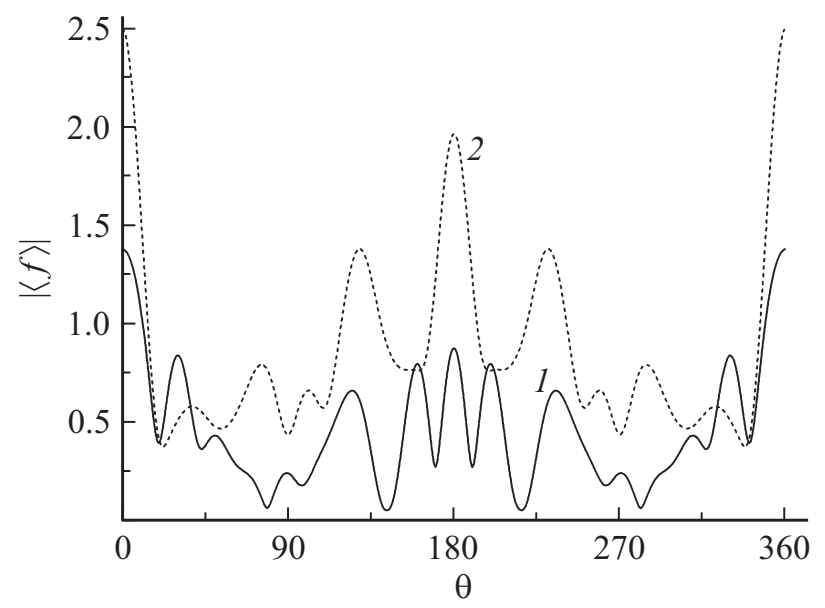

Pис. 4. Угловая зависимость усредненной диаграммы для тела с сечением в виде снежинки Коха. Кривые 1 и 2 соответствуют 1- и 2-й итерации.

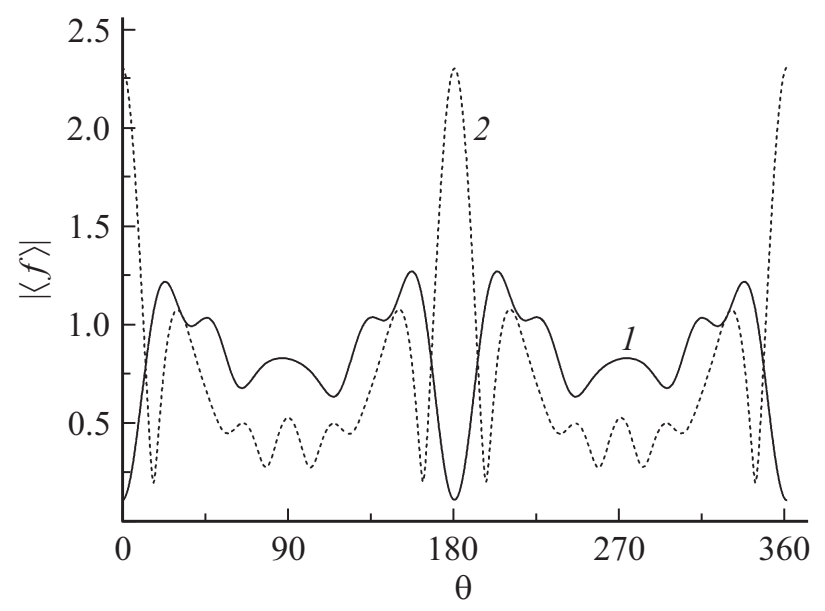

Рис. 5. Угловая зависимость усредненной диаграммы для тела в виде кривой Серпинского. Кривые 1 и 2 соответствуют 1- и 2-й итерации.

В последних двух случаях максимальный размер сечения тела по оси $x$ был равен $2 k a=20$. Геометрии указанных фракталоподобных тел приведены на рис. 1 и 2. Число точек коллокации на одной длине волны для всех шести форм сечения тела составляло $N_{\lambda}=30$. Число угловых гармоник было выбрано $Q=24$. Плоская волна падала перпендикулярно оси тела. Как следует из таблицы, относительная разность правой и левой части равенства $(19)$, т. е. величина $\Delta_{\text {ot }}$ не превосходит $5 \cdot 10^{-5}$. Таким образом, задача дифракции решается на основе МПГУ с высокой точностью.

Рассмотрим результаты, касающиеся расчета усредненной по углам падения волны диаграммы рассеяния. В качестве теста мы сравнили значения усредненной диаграммы сферы, полученные при помощи МПГУ и при помощи точной формулы (39). Радиус сферы был равен $k a=10$. Угловые зависимости модуля усредненной диаграммы приведены на рис. 3. Кривая 1 на рисунке иллюстрирует точное значение усредненной диаграммы, полученной из (39), а кривые 2 и 3 относятся к случаю, когда величина параметра $k \delta$ была равна $10^{-3}$ и $10^{-4}$ соответственно. Как видно, с уменьшением параметра $\delta$ приближенное решение приближается к точному.

На рис. 4 и 5 изображены угловые зависимости усредненной диаграммы рассеяния для частицы с сечением в виде снежинки Коха и в виде кривой Серпинского соответственно. Максимальный размер сечения тела по оси $x$ был равен $2 k a=20$. Параметр $k \delta$ был равен $10^{-3}$. Следует отметить, что если в двумерном случае вид усредненной диаграммы рассеяния позволял составить „некое первое представление“ о геометрии рассеивателя [1], то в трехмерном случае столь очевидного соответствия не наблюдается, по-видимому, из-за его более сложной геометрии.

\section{Финансирование работы}

Работа выполнена при частичной поддержке Российского фонда фундаментальных исследований (проекты № 19-02-00654, 18-02-00961).

\section{Список литературы}

[1] Кюркчан А.Г., Смирнова Н.И. // РЭ. 2017. Т. 62. № 5. C. 476; Kyurkchan A.G., Smirnova N.I. // J. Commun. Technol. Electron. 2017. V. 62. N 5. P. 502.

[2] Кюркчан А.Г., Смирнова Н.И. Математическое моделирование в теории дифракции с использованием априорной информации об аналитических свойствах решения. М: ИД Медиа Паблишер, 2014. 226 c.; Kyurkchan A.G., Smirnova N.I. Mathematical Modeling in Diffraction Theory Based on A Priori Information on the Analytical Properties of the Solution. Amsterdam: Elsevier, 2016.

[3] Mishchenko M.I., Zakharova N.T., Khlebtsov N.G., Videen G., Wriedt T. // J. Quant. Spectr. Rad. Trans. 2017. V. 202. P. 240.

[4] Kyurkchan A.G., Smirnova N.I. // J. Quant. Spectr. Rad. Trans. 2014. V. 146. P. 304.

[5] Waterman P.C. // Proc. IEEE. 1965. V. 53. P. 805.

[6] Кюркчан А.Г., Смирнова Н.И., Чиркова А.П. // Т-Comm. (Технологии информационного общества). 2013. № 11. C. 119; Kyurkchan A.G., Smirnova N.I., Chirkova A.P. // TComm.(Technol. Inform.-Oriented Soc.). 2013. N 11. P. 119.

[7] Кюркчан А.Г., Смирнова Н.И., Чиркова А.П. // РЭ. 2015. T. 60. № 3. C. 247; Kyurkchan A.G., Smirnova N.I., Chirkova A.P. // J. Commun. Technol. Electron. 2015. V. 60. N 3. P. 232.

[8] Кюркчан А.Г., Анютин А.П. // ДАН. 2002. Т. 385. № 3. C. 309; Kyurkchan A.G., Anyutin A.P. // Doklady Mathematics. 2002. V. 66. № 1. P. 132.

[9] Борн М., Воль $\varnothing$ Э. Основы оптики. М.: Наука, 1970. 856 с.; Born M., Wolf E. Principles Of Optics. N. Y.: Pergamon Press, 1964.

[10] Маненков С.A. // РЭ. 2018. Т. 63. № 1. С. 3; Manenkov S.A. // J. Commun. Technol. Electron. 2018. V. 63. № 1. P. 1. 
[11] Борен К., Хафмен Д. Поглощение и рассеяние света малыми частицами. М.: Мир, 1986. 664 c.; Bohren K.F., Huffman D.R. Absorption and Scattering of Light by Small Particles. N. Y.: John Wiley \& Sons, 1983.

[12] Справочник по специальным функциям с формулами, графиками и математическими таблицами / Под ред. Абрамовица М., Стиган И.М.: Наука, 1979. 832 с.; Handdook of Mathematical Functions with Formulas, Graphs and Mathematical Tables. Edited by Abramowitz M. and Stegun I.A. National Bureau of Standards, 1964.

[13] Маненков С.A. // Акустический журнал. 2014. Т. 60. № 2. C. 129; Manenkov S.A. // Acoustical Physics. 2014. V. 60. N 2. P. 127.

[14] Кроновер Р.М. Фракталы и хаос в динамических системах. Основы теории. М.: Постмаркет, 2000. 352 с.; Crownover R.M. Intoduction to Fractals and Chaos. Boston: Jones and Bartlett Publishers, 1995. 